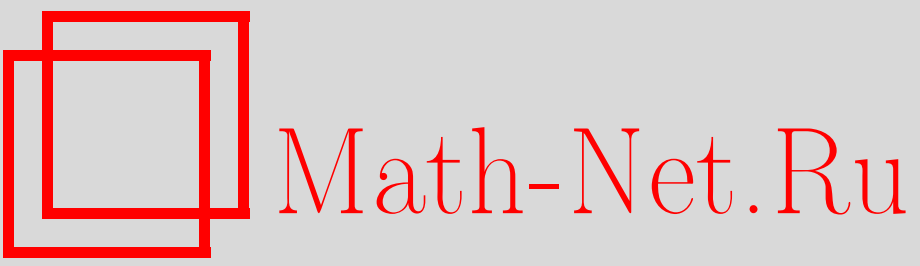

С. П. Горшков, Исследование некоторых подклассов мультиаффинных, биюнктивных, слабо положительных и слабо отрицательных булевых функций, $M a-$ тем. вопр. криптогр., 2016, том 7, выпуск 4, 51-66

DOI: https://doi.org/10.4213/mvk203

Использование Общероссийского математического портала Math-Net.Ru подразумевает, что вы прочитали и согласны с пользовательским соглашением

http://www. mathnet.ru/rus/agreement

Параметры загрузки:

IP: 3.80 .181 .102

26 апреля 2023 г., 09:49:29

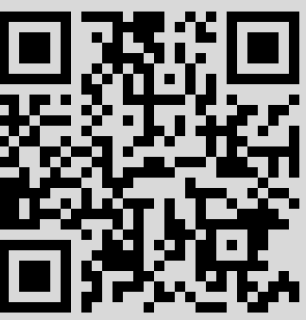


МАТЕМАТИЧЕСКИЕ ВОПРОСЫ КРИПТОГРАФИИ

2016 T. 7 № 4 C. 51-66

УДК 519.716.39+519.719.2

\title{
Исследование некоторых подклассов мультиаффинных, биюнктивных, слабо положительных и слабо отрицательных булевых функций
}

\author{
С. П. Горшков
}

Академия криптографии Российской Федераџии, Москва

Получено 30.V.2016

Аннотация. Множества мультиаффинных (обозначается $A$ ), биюнктивных (или 2-КНФ, $B i$ ), слабо положительных (или антихорновских, $W P$ ) и слабо отрицательных (или хорновских, $W N$ ) булевых функций порождают классы полиномиально решаемых систем уравнений. Изучены классы функций $A \cap B, B i \cap B$, где $B$ - множество бент-функций. Описаны множества возможных значений алгебраической степени нелинейности функций из классов $A, B i, W P, W N$. Рассмотрены вопросы построения функций из классов $W P, W N$ на основе функций от меньшего числа переменных.

Ключевые слова: бент-функции, мультиаффинные булевы функции, 2-КНФ, хорновские булевы функции

Investigation of some subclasses of multiaffine, bijunctive, weakly positive and weakly negative Boolean functions

\section{S. P. Gorshkov}

Academy of Cryptography of the Russian Federation, Moscow

Abstract. Sets of multiaffine (denoted by $A$ ), bijunctive (2-CNF, $B i$ ), weakly positive (or anti-Horn, $W P$ ) and weakly negative (or Horn, $W N$ ) Boolean functions generate classes of polynomially solvable systems of equations. We investigate functional classes $A \cap B, B i \cap B$, where $B$ is the set of bent functions. Sets of possible values of algebraic nonlinearity degree of functions from $A, B i, W P, W N$ are described. Problems of construction of functions from classes $W P, W N$ by means of functions of smaller number of variables are considered.

Keywords: bent functions, multiaffine functions, 2-CNF, Horn Boolean functions

Citation: Mathematical Aspects of Cryptography, 2016, v. 7, № 4, pp. 51-66 (Russian)

(c) Академия криптографии Российской Федерации, 2016 г. 


\section{Основные обозначения и определения:}

$N$ - множество натуральных чисел,

$V_{n}-n$-мерное пространство булевых векторов, $n \in N$,

$F_{n}$ - множество булевых функций от $n$ переменных,

$\operatorname{Sym}(n)$ - симметрическая группа степени $n$,

$S_{n}$ - симметрическая группа перестановок координат векторов из $V_{n}$, подгруппа $\operatorname{Sym}\left(2^{n}\right)$, изоморфна $\operatorname{Sym}(n)$,

$\Sigma_{n}$ - группа сдвигов, является подгруппой $S_{2^{n}}$, состоит из преобразований $\tau_{\beta}(x)=x \oplus \beta$, где $x, \beta \in V_{n}$,

$Q_{n}$ - группа Джевонса, являющаяся полупрямым произведением групп $S_{n}$ и $\Sigma_{n}$, состоит из преобразований вида

$$
s_{\pi, \beta}(x)=\tau_{\beta}(\pi(x)), \text { где } \tau_{\beta} \in \Sigma_{n}, \pi \in S_{n},
$$

$\left[f\left(x_{1}, \ldots, x_{n}\right)\right]_{\left\langle S_{n}\right\rangle}-$ класс эквивалентности булевых функций относительно группы $S_{n}$ с представителем $f\left(x_{1}, \ldots, x_{n}\right)$,

$\left[f\left(x_{1}, \ldots, x_{k}\right)\right]_{\left\langle Q_{k}\right\rangle}-$ класс эквивалентности булевых функций относительно перестановок группы Джевонса с представителем $f$,

$\operatorname{deg}\left(f\left(x_{1}, \ldots, x_{n}\right)\right)$ - алгебраическая степень нелинейности функции $f$.

Вектор $\left(\alpha_{1}, \ldots, \alpha_{n}\right) \in V_{n}$ назовем выполняющим вектором функции $f\left(x_{1}, \ldots, x_{n}\right)$, если $f\left(\alpha_{1}, \ldots, \alpha_{n}\right)=1$. Множество всех выполняющих векторов функции $f$ обозначим $E_{f}$. Значение $\|f\|=\left|E_{f}\right|$ называется весом функции.

О п р е д е л е н и е 1 . Булева функция $f\left(x_{1}, \ldots, x_{n}\right)$ называется:

1) мультиаффинной, если существует представление функции $f$ в виде конъюнкции аффинных функций:

$$
f \equiv \wedge_{i=1}^{t}\left(\alpha_{i 1} x_{1} \oplus \cdots \oplus a_{i m} x_{n} \oplus a_{i 0}\right),
$$

где $a_{i j} \in\{0,1\}, \quad i=1, \ldots, t, \quad j=0, \ldots, n$,

2) биюнктивной, если $f \equiv 1$ или существует представление функции $f$ в виде следующей конъюнктивной нормальной формы (КНФ):

$$
f \equiv\left(\stackrel{t}{\wedge} x_{i=1}^{\alpha_{i}}\right) \wedge\left(\bigwedge_{j=1}^{q}\left(x_{s_{j 1}}^{\beta_{j 1}} \vee x_{s_{j 2}}^{\beta_{j 2}}\right)\right)
$$

где $\alpha_{i}, \beta_{j 1}, \beta_{j 2} \in\{0,1\}, s_{i}, s_{j 1}, s_{j 2} \in\{1, \ldots, k\}, \quad i=1, \ldots, t, \quad j=$ $1, \ldots, q$, 
3) слабо положительной (или антихорновской), если $f \equiv 1$ или существует представление $f$ в виде следующей КНФ:

$$
f \equiv \bigwedge_{i=1}^{t}\left(x_{s_{i 1}}^{\alpha_{i}} \vee x_{s_{i 2}} \vee \ldots \vee x_{s_{i n_{i}}}\right)
$$

где $\alpha_{i} \in 0,1, \quad\left\{n_{i}\right\} \leqslant n,\left\{s_{i j}\right\} \in\{1, \ldots, n\}, j=1, \ldots, n_{i}, i=1, \ldots t$,

4) слабо отрищательной или хорновской, если $f \equiv 1$ или существует представление $f$ в виде следующей КНФ:

$$
f \equiv x \wedge_{i=1}^{t}\left(x_{s_{i 1}}^{\alpha_{i}} \vee \bar{x} s_{i 2} \vee \ldots \vee \bar{x}_{s_{i n_{i}}}\right)
$$

где $\alpha_{i} \in\{0,1\}, n_{i} \leqslant n, s_{i j} \in\{1, \ldots, n\}, j=1, \ldots, n_{i}, i=1, \ldots, t$.

Множества всех функций соответственно классов 1) - 4) обозначим $A, B i$, $W P, W N$. Формулы (1), (2), (3), (4) для функций классов $A, B i, W P, W N$ соответственно будем называть приведенныли представлениями.

Классы функций $A, B i, W P, W N$ будем еще называть классами Шефера.

Критерий слабой положительности (см. [2]): функция $f\left(x_{1}, \ldots, x_{n}\right) \not \equiv 0$ является слабо положительной, если и только если для любых двух выполняющих векторов $\{\alpha, \beta\} \subseteq E_{f}$ этой функции справедливо включение

$$
(\alpha \vee \beta) \in E_{f} .
$$

Если для функций $f\left(x_{1}, \ldots, x_{n}\right), h\left(x_{1}, \ldots, x_{n}\right)$ выполняется равенство $f \cdot h \equiv f$, то $h$ называется имплицентой функции $f$.

\section{1. О пересечении классов мультиаффинных и биюнктивных функций с классом бент-функций}

Напомним некоторые понятия [3].

Скалярное произведение $\langle x, y\rangle$ векторов $x=\left(x_{1}, \ldots, x_{n}\right) \in V_{n}, y=$ $\left(y_{1}, \ldots, y_{n}\right) \in V_{n}$ находится по формуле

$$
\langle x, y\rangle=\bigoplus_{i=1}^{n} x_{i} y_{i}
$$

Для всякого $\alpha=\left(\alpha_{1}, \ldots, \alpha_{n}\right) \in V_{n}$ определим величину

$$
\Delta_{\alpha}^{f}=2^{n-1}-\|f(x) \oplus\langle\alpha, x\rangle\|,
$$


где $\|f(x) \oplus\langle\alpha, x\rangle\|-$ вес функции $f(x) \oplus\langle\alpha, x\rangle$.

Набор $\left\{\Delta_{\alpha}^{f} \mid \alpha \in V_{n}\right\}$ называется статистической структурой функции $f\left(x_{1}, \ldots, x_{n}\right)$. Числа $\Delta_{\alpha}^{f}$ называются коэффициентами статистической структуры функции. При этом

$$
\Delta_{0}^{f}=2^{n-1}-\|f(x)\| \text { и }\|f(x)\|=2^{n-1}-\Delta_{0}^{f} .
$$

Функция $f\left(x_{1}, \ldots, x_{n}\right)$ называется бент-функцией ([3, с. 260]), если

$$
\left|\Delta_{\alpha}^{f}\right|=2^{\frac{n}{2}-1} \text { для всех } \alpha \in V_{n} .
$$

Ясно, что необходимым условием того, что $f-$ бент-функция, является четность $n$. Известно, что для всякого четного $n$ существуют бент-функции. Если $f\left(x_{1}, \ldots, x_{n}\right)$ - бент-функция, то ее вес может принимать только два значения:

$$
\|f\|=2^{n-1}+2^{\frac{n}{2}-1} \text { или }\|f\|=2^{n-1}-2^{\frac{n}{2}-1},
$$

т. е. бент-функции не являются равновероятными. Всякая бент-функция существенно зависит от всех своих переменных. Если $f(x) \equiv f\left(x_{1}, \ldots, x_{n}\right)-$ бент-функция и $L$ - невырожденное аффинное преобразование $V_{n}$, то $f(x L)$ - бент-функция.

Символом $B$ обозначим множество бент-функций, $B_{n}-$ множество бентфункций, зависящих от $n$ переменных.

Теорема 1. Справедливо соотношение

$$
A \cap B=\left[x_{1} \cdot x_{2}\right]_{\left\langle Q_{2}\right\rangle} .
$$

Доказательство. Легко показать, что $A \cap B_{2}=\left[x_{1} \cdot x_{2}\right]_{\left\langle Q_{2}\right\rangle}$.

Предположим, что существует мультиаффинная бент-функция $f\left(x_{1}, \ldots, x_{n}\right)$, зависящая от $n \geqslant 4$ переменных ( $n-$ четное). Так как $f \in A$, то $\|f\|=2^{r}, 0 \leqslant r \leqslant n-1$ (заметим, что $f$ существенно зависит от $n$ переменных). С другой стороны, $f \in B$, поэтому $\|f\|=2^{n-1}-2^{\frac{n}{2}-1}$. Выполняется равенство $2^{n-1}-2^{\frac{n}{2}-1}=2^{r}$, или

$$
2^{n-1}=2^{\frac{n}{2}-1}+2^{r} \text {. }
$$

Нетрудно видеть, что $\frac{n}{2}-1<r<n-1$. Из (5) следует, что

$$
2^{\frac{n}{2}}=1+2^{r-\frac{n}{2}+1} \text {. }
$$


Слева в (6) четное число, а справа - нечетное. Полученное противоречие заканчивает доказательство теоремы.

Перейдем к рассмотрению множества $B i \cap B$.

В [4] доказано, что если $n \geqslant 5, f \in B i_{n}$ и $f$ существенно зависит от $n$ переменных, то

$$
\|f\|=0,5 \cdot 2^{n}+1 \text { или }\|f\|<0,5 \cdot 2^{n} .
$$

Существенно используя доказательство этой теоремы, получим более точную оценку.

Лемма 1. Если $n \geqslant 8, f \in B i_{n}$ u $f$ существенно зависит от $n$ переменHblx, mo

$$
\|f\|=2^{n-1}+1 \text { или }\|f\|<0,44 \cdot 2^{n} .
$$

Доказательство. По условию леммы $f \not \equiv 0$ и существует $\left(\alpha_{1}, \ldots, \alpha_{n}\right) \in E_{f}$. Рассмотрим функцию $g\left(x_{1}, \ldots, x_{n}\right) \equiv f\left(x_{1} \oplus \alpha_{1} \oplus 1, \ldots, x_{n} \oplus \alpha_{n} \oplus 1\right)$. Для этой функции имеем: $g(1, \ldots, 1)=1$, поэтому $g \in B i \cap W P$. Кроме того, $\|g\|=\|f\|$. Далее считаем $f \in(B i \cap W P)$.

Пусть (2) - минимальная КНФ для $f$. Если $f$ имеет имплиценту вида $x_{i}^{\beta}$, то $\|f\| \leqslant 2^{n-1-1}=\frac{1}{4} \cdot 2^{n}$ (если есть еще одна имплицента вида $x_{j}^{\delta}$ ) или $\|f\| \leqslant 3 \cdot 2^{n-1-2}=\frac{3}{8} \cdot 2^{n}$ (если есть имплицента вида $x_{j} \vee x_{l}^{\lambda}$ ), что соответствует (7).

Далее считаем, что $f$ не имеет имплицент вида $x_{i}^{\beta}$, а каждая из переменных $x_{2}, \ldots, x_{n}$ в (2) входит не большее число раз, чем $x_{1}$. Без ограничения общности можем считать, что (2) имеет вид

$$
f \equiv \stackrel{\wedge}{i=1}_{i=1}\left(x_{1} \vee x_{i+1}^{\beta_{i}}\right) \wedge \stackrel{\curlywedge}{t}_{j=1}\left(\bar{x}_{1} \vee x_{s_{j}}\right) \wedge f^{\prime}\left(x_{2}, \ldots, x_{n}\right),
$$

где $p \geqslant 0, t \geqslant 0$ (в случае $p=0$ или $t=0$ считаем, что соответствующие сомножители отсутствуют).

Обозначим $X_{1}=\left\{x_{2}, \ldots, x_{p}\right\}, X_{2}=\left\{x_{s_{1}}, \ldots, x_{s_{t}}\right\}$. Предположим, что $X_{1} \cap X_{2} \neq \varnothing, \quad x_{2} \in X_{1} \cap X_{2}$. Запись (8) не может содержать произведение $\left(x_{1} \vee x_{2}\right)\left(\bar{x}_{1} \vee x_{2}\right)$, так как $\left(x_{1} \vee x_{2}\right)\left(\bar{x}_{1} \vee x_{2}\right) \equiv x_{2}$. Пусть $f$ содержит произведение $\left(x_{1} \vee \bar{x}_{2}\right)\left(\bar{x}_{1} \vee x_{2}\right)$, при этом $\left(x_{1} \vee \bar{x}_{2}\right)\left(\bar{x}_{1} \vee x_{2}\right) \equiv\left(x_{1} \oplus x_{2} \oplus 1\right)$. Если $f^{\prime}$ имеет элементарную имплиценту вида $\left(x_{r_{1}} \vee x_{r_{2}}^{\delta}\right)$, где $r_{1}>2, r_{2}>2$, то $\|f\| \leqslant 2 \cdot 3 \cdot 2^{n-2-2}=\frac{3}{8} \cdot 2^{n}$ и лемма верна. В случае когда в $f^{\prime}$ имеется элементарная имплицента с переменной $x_{2}$, опять просто показывается, что $\|f\| \leqslant \frac{3}{8} \cdot 2^{n}$. 
Далее считаем, что $X_{1} \cap X_{2}=\varnothing$. Рассмотрим случай $f^{\prime} \equiv 1$, тогда $X_{1} \cup$ $X_{2}=\left\{x_{2}, \ldots, x_{n}\right\}$. При этом

$$
\|f\|=2^{p}+2^{n-p} .
$$

Если $p=0$ или $p=n-1$, то

$$
\|f\|=1+2^{n-1} .
$$

В случае $1 \leqslant p<n-1, n \geqslant 6$

$$
\|f\| \leqslant 2+2^{n-2}<0,3 \cdot 2^{n} .
$$

Ниже полагаем $X_{1} \cap X_{2}=\varnothing, f^{\prime} \not \equiv 1$. Пусть $p=n-1, t=0$. Тогда $f$ имеет имплиценту вида

$$
f_{1} \equiv\left(\stackrel{\wedge}{i=1}_{n-1}^{\wedge}\left(x_{1} \vee x_{i+1}^{\beta_{i}}\right)\right) \wedge\left(x_{m}^{\delta} \vee x_{r}^{\varepsilon}\right), \quad \text { где } m \geqslant 2, \quad r \geqslant 2 .
$$

В этом случае $\left\|f_{1}\right\| \leqslant 2^{n-1}+1-2^{n-3}$ и $\|f\| \leqslant\left\|f_{1}\right\|<0,4 \cdot 2^{n}$ при $n \geqslant 6$. Аналогично рассматривается случай $p=0, t=n-1$. Если $p+t=n-1$, $p \geqslant 1, t \geqslant 1$, то верно (9).

Далее считаем, что $p+t \leqslant n-2$.

1) $p \geqslant 1, t \geqslant 1, p+t \geqslant 3$. В этом случае

$$
\|f\| \leqslant\left\|\left(x_{1} \vee x_{2}\right)\left(x_{1} \vee x_{3}\right)\left(\bar{x}_{1} \vee x_{4}\right)\right\|=6 \cdot 2^{n-4}=0,375 \cdot 2^{n} ;
$$

здесь функцию $\left(x_{1} \vee x_{2}\right)\left(x_{1} \vee x_{3}\right)\left(\bar{x}_{1} \vee x_{4}\right)$ рассматриваем как функцию от $n$ переменных.

2) $p \geqslant 1, t \geqslant 1, p+t<3$ или $p=t=1$. При таких условиях каждая переменная в (2) встречается не более двух раз. Так как функция $f$ существенно зависит не менее чем от четырех переменных, то найдутся переменные $x_{4}, x_{5}$, которые не содержатся в $X_{1}, X_{2}$. Рассмотрим все возможные случаи:

$$
\begin{gathered}
\|f\| \leqslant\left\|\left(x_{1} \vee x_{2}\right)\left(\bar{x}_{1} \vee x_{3}\right)\left(x_{4} \vee x_{5}\right)\right\|=\frac{3}{8} \cdot 2^{n}=0,375 \cdot 2^{n}, \\
\|f\| \leqslant\left\|\left(x_{1} \vee x_{2}\right)\left(\bar{x}_{1} \vee x_{3}\right)\left(x_{2} \vee x_{4}\right)\left(x_{3} \vee x_{5}\right)\right\|=\frac{3}{8} \cdot 2^{n}=0,375 \cdot 2^{n}, \\
\|f\| \leqslant\left\|\left(x_{1} \vee x_{2}\right)\left(\bar{x}_{1} \vee x_{3}\right)\left(\bar{x}_{2} \vee x_{4}\right)\left(x_{3} \vee x_{5}\right)\right\|=\frac{9}{32} \cdot 2^{n}<0,3 \cdot 2^{n}, \\
\|f\| \leqslant\left\|\left(x_{1} \vee x_{2}\right)\left(\bar{x}_{1} \vee x_{3}\right)\left(\bar{x}_{2} \vee x_{4}\right)\left(\bar{x}_{3} \vee x_{5}\right)\right\|=\frac{3}{16} \cdot 2^{n}<0,2 \cdot 2^{n} .
\end{gathered}
$$


3) $p=1, t=0$ (случай $p=0, t=1$ рассматривается аналогично). Так как $x_{1}$ в (2) встречается один раз, а все остальные переменные встречаются не чаще, чем $x_{1}$, то все переменные в (2) встречаются по одному разу. Так как $n \geqslant 6$, то

$$
\|f\| \leqslant\left\|\left(x_{1} \vee x_{2}\right)\left(x_{3} \vee x_{4}\right)\left(x_{5} \vee x_{6}\right)\right\|=\left(\frac{3}{4}\right)^{3} \cdot 2^{n}<0,43 \cdot 2^{n}
$$

4) $p=2, t=0$. Если некоторая переменная входит в (2) в разных степенях, то доказательство сводится к случаям 1) или 2). Далее считаем, что все переменные входят в формулу не более двух раз и что каждая переменная входит в одной степени. Так как переменные, входящие в нулевой степени (литералы вида $\bar{x}_{i}$ ), можно инвертировать, то без ограничения общности можно считать, что функция $f$ является монотонной.

Пусть $r$ переменных входят в (2) по два раза, а $l$ переменных входят в (2) по одному разу $(r+l=n)$. Всего вхождений переменных $2 r+l$, а число элементарных сомножителей в (2) равно $v=\frac{2 r+l}{2}=r+\frac{l}{2}$ (отсюда, в частности, следует, что $l \geq 0$ - четное число). Если $l=0$, то $v=n \geqslant 8$. Выберем в (2) сомножитель $\left(x_{1} \vee x_{2}\right)$ и удалим из (2) сомножители, содержащие $x_{1}$ или $x_{2}$. Всего сомножителей останется $v-3$. Выберем еще один сомножитель вида $\left(x_{3} \vee x_{4}\right)$, после этого можем выбрать еще один сомножитель вида $\left(x_{5} \vee x_{6}\right)$ и в итоге попадаем в условия п. 3). Нетрудно показать, что и в остальных случаях можно выбрать три сомножителя от разных переменных.

5) $3 \leqslant p \leqslant n-2, t=0$. При этом возможны два варианта. В первом случае функция $f$ имеет имплиценту вида $\stackrel{\wedge}{i=1}_{i}^{p}\left(x_{1} \vee x_{i+1}^{\beta_{i}}\right) \wedge\left(x_{p} \vee x_{p+1}\right)$. Тогда (учитываем, что $p \geqslant 3$ )

$$
\|f\| \leqslant \frac{3}{4} \cdot 2^{n-p-1} \cdot\left(2^{p}+1\right) \leqslant \frac{27}{64} \cdot 2^{n}<0,43 \cdot 2^{n} .
$$

Во втором случае функция $f$ имеет одну из двух имплицент:

5.1) $\left(x_{1} \vee x_{2}\right)\left(x_{1} \vee x_{3}\right)\left(x_{1} \vee x_{4}\right)\left(x_{2} \vee x_{5}\right)-$ тогда

$\|f\| \leqslant\left\|\left(x_{1} \vee x_{2}\right)\left(x_{1} \vee x_{3}\right)\left(x_{1} \vee x_{4}\right)\left(x_{2} \vee x_{5}\right)\right\|=\frac{14}{32} \cdot 2^{n}<0,44 \cdot 2^{n}$.

5.2) $\left(x_{1} \vee x_{2}\right)\left(x_{1} \vee x_{3}\right)\left(x_{1} \vee x_{4}\right)\left(\bar{x}_{2} \vee x_{5}\right)$ - тогда

$\|f\| \leqslant\left\|\left(x_{1} \vee x_{2}\right)\left(x_{1} \vee x_{3}\right)\left(x_{1} \vee x_{4}\right)\left(\bar{x}_{2} \vee x_{5}\right)\right\|=\frac{13}{32} \cdot 2^{n}<0,41 \cdot 2^{n}$.

Лемма доказана. 
Замечание 1. А. В. Тарасов сообщил автору о полученном им более сильном утверждении: если биюнктивная функция $f\left(x_{1}, \ldots, x_{n}\right)$ существенно зависит от всех $n \geqslant 5$ переменных, то либо $\left\|f\left(x_{1}, \ldots, x_{n}\right)\right\|=2^{n-1}+1$, либо $\left\|f\left(x_{1}, \ldots, x_{n}\right)\right\| \leqslant 0,375 \cdot 2^{n-1}+3$.

Теорема 2. Справедливо соотношение

$$
B i \cap B=\left[x_{1} \cdot x_{2}\right]_{\left\langle Q_{2}\right\rangle} \cup\left[x_{1} \vee x_{2}\right]_{\left\langle Q_{2}\right\rangle} .
$$

Доказательство. Достаточно ограничиться рассмотрением только представителей биюнктивных функций в классе эквивалентности относительно группы Джевонса, причем таким представителем всегда может быть функция из множества $B i \cap W P$.

При $n=2$ имеем $B i_{2} \cap B_{2}=\left[x_{1} \cdot x_{2}\right]_{\left\langle Q_{2}\right\rangle} \cup\left[x_{1} \vee x_{2}\right]_{\left\langle Q_{2}\right\rangle}$.

Для $n=4$ достаточно рассмотреть только представителей биюнктивных функций в классе эквивалентности относительно группы Джевонса, имеющих вес 6 или 10 и существенно зависящих от всех переменных. Согласно [2], таких функций восемь. Все они не являются бент-функциями.

Для $n=6$ достаточно рассмотреть только представителей биюнктивных функций в классе эквивалентности относительно группы Джевонса, имеющих вес 28 или 36 и существенно зависящих от всех переменных. А. В. Тарасовым с помощью компьютера были найдены все биюнктивные функции от 6 переменных. Среди них выявлено 5 функций, существенно зависящих от 6 переменных и имеющих вес 28 или 36. Все они не являются бент-функциями.

Перейдем к рассмотрению случая $n \geqslant 8$. Бент-функция может иметь вес

$$
\|f\|=2^{n-1}+2^{\frac{n}{2}-1} \text { или }\|f\|=2^{n-1}-2^{\frac{n}{2}-1} \text {. }
$$

Ввиду леммы при $n \geqslant 8$ биюнктивная функция не может иметь вес $\|f\|=$ $2^{n-1}+2^{\frac{n}{2}-1}$.

Заметим, что если $n \geqslant 8, f \in B_{n}$ и $\|f\|=2^{n-1}-2^{\frac{n}{2}-1}$, то

$$
\|f\| \geqslant 0,46 \cdot 2^{n} \text {. }
$$

Это свойство следует из очевидных выкладок: при $n \geqslant 8$

$$
\|f\|=2^{n-1}-2^{\frac{n}{2}-1}=2^{n} \cdot\left(\frac{1}{2}-\frac{1}{2^{\frac{n}{2}+1}}\right) \geqslant 2^{n} \cdot\left(\frac{1}{2}-\frac{1}{2^{\frac{8}{2}+1}}\right)>0,46 \cdot 2^{n},
$$

т. е. $0,46 \cdot 2^{n}<\|f\|<0,5 \cdot 2^{n}$. Но если $f-$ биюнктивная функция, существенно зависящая от $n \geqslant 8$ переменных, и $\|f\| \neq 2^{n-1}+1$, то $\|f\|<0,44 \cdot 2^{n}$. Поэтому $B i_{n} \cap B_{n}=\varnothing$ при $n \geqslant 8$. Теорема доказана. 
Для пересечений класса бент-функций с классами слабо положительных и слабо отрицательных функций приведем лишь следующие простые утверждения.

Утверждение 1. Булева функиия $f\left(x_{1}, \ldots, x_{n}\right)$ принадлежит $W P \cap B$ тогда и только тогда, когда $f\left(\bar{x}_{1}, \ldots, \bar{x}_{n}\right) \in W N \cap B$.

Утверждение 2. Верно соотношение

$$
W P \cap B \cap F_{2}=\left[x_{1} \cdot x_{2}\right]_{\left\langle Q_{2}\right\rangle} \cup\left[x_{1} \vee x_{2}\right]_{\left\langle S_{2}\right\rangle} \cup\left[x_{1} \vee \bar{x}_{2}\right]_{\left\langle S_{2}\right\rangle} .
$$

Утверждение 3. Верны соотношения

$$
\bar{x}_{1} \bar{x}_{2} \oplus\left(x_{3} \vee x_{4}\right) \in W P \cap B, \quad x_{1} x_{2} \oplus x_{3} x_{4} \oplus 1 \in W N \cap B .
$$

\section{2. Об алгебраической степени нелинейности функций из классов Шефера}

Утверждение 4. При любом $n \geqslant 1$ и любом $r \in\{1, \ldots, n\}$ существует такая мультиаффинная функиия $f\left(x_{1}, \ldots, x_{n}\right)$, существенно зависящая от всех переменных, что $\operatorname{deg} f=r$.

Доказательство. При $r=1$ искомая функция имеет вид $f \equiv x_{1} \oplus \ldots \oplus x_{n}$. В случае $r \geqslant 2$ функция $f \equiv\left(x_{1} \oplus \ldots \oplus x_{n-r}\right) \cdot x_{n-r+1} \cdot \ldots \cdot x_{n}$ удовлетворяет условиям теоремы.

\section{Теорема 3.}

1) При $n \geqslant 3$ не существует биюнктивных (слабо положительных, слабо отрицательных) булевых функций, существенно зависящих от всех переменных и имеющих алгебраическую степень нелинейности 1.

2) При любом $n \geqslant 2$ и любом $r, \frac{n}{2} \leqslant r \leqslant n$, существует такая биюнктивная (слабо положительная, слабо отрицательная) функция $f\left(x_{1}, \ldots, x_{n}\right)$, существенно зависящая от всех переменных, что $\operatorname{deg} f=r$.

Доказательство. Предположим, что для некоторого $n \geqslant 3$ существует биюнктивная функция $f\left(x_{1}, \ldots, x_{n}\right)$, существенно зависящая от всех переменных и имеющая алгебраическую степень нелинейности 1. Тогда функция $f$ имеет вид $f\left(x_{1}, \ldots, x_{n}\right)=x_{1} \oplus \ldots \oplus x_{n} \oplus \alpha$. Если $n=3$, то $f\left(x_{1}, x_{2}, x_{3}\right)=x_{1} \oplus x_{2} \oplus x_{3} \oplus \alpha$. При любом $\alpha \in\{0,1\}$ эта функция не является биюнктивной, поскольку для нее не выполняется критерий биюнктивности $[1,6]$. 
В случае $n>3$ рассмотрим функцию $g\left(x_{1}, x_{2}, x_{3}\right)=f\left(x_{1}, x_{2}, x_{3}, 0, \ldots, 0\right)$. $\mathrm{C}$ одной стороны, функция $g$ биюнктивна как подфункция биюнктивной функции, но функция $g$ имеет вид $g\left(x_{1}, x_{2}, x_{3}\right)=x_{1} \oplus x_{2} \oplus x_{3} \oplus \beta$, поэтому $g \notin B i, f \notin B$. Доказательство первой части теоремы для слабо положительных и слабо отрицательных функций проводится аналогично, при этом используется свойство $\left(x_{1} \oplus x_{2} \oplus x_{3} \oplus \alpha\right) \notin(W P \cup W N)$, где $\alpha \in\{0,1\}$.

Пусть $n \geqslant 2$ и $\frac{n}{2} \leqslant r \leqslant n$. При $r=\frac{n}{2}$ рассмотрим функцию

$$
f\left(x_{1}, \ldots, x_{n}\right) \equiv\left(x_{1} \oplus x_{2} \oplus 1\right)\left(x_{3} \oplus x_{4} \oplus 1\right) \ldots\left(x_{2(n-r)-1} \oplus x_{2(n-r)} \oplus 1\right) .
$$

В случае $r=n$ полагаем

$$
f\left(x_{1}, \ldots, x_{n}\right) \equiv x_{1} \cdot \ldots \cdot x_{n},
$$

а при $\frac{n}{2}<r<n$ рассмотрим функцию

$$
\begin{aligned}
& f\left(x_{1}, \ldots, x_{n}\right) \equiv \\
& \equiv\left(x_{1} \oplus x_{2} \oplus 1\right)\left(x_{3} \oplus x_{4} \oplus 1\right) \ldots\left(x_{2(n-r)-1} \oplus x_{2(n-r)} \oplus 1\right) \cdot x_{2(n-r)+1} \cdot \ldots \cdot x_{n} .
\end{aligned}
$$

Так как $\left(x_{1} \oplus x_{2} \oplus 1\right) \equiv\left(x_{1} \vee \bar{x}_{2}\right) \cdot\left(\bar{x}_{1} \vee x_{2}\right)$, то $f \in(B i \cap W P \cap W N)$. Функция $f$ существенно зависит от всех переменных. Алгебраическая степень нелинейности функции $f$ равна

$$
\operatorname{deg} f=n-r+(n-2(n-r))=r .
$$

Теорема доказана.

\section{3. О построении слабо положительных и слабо отрицательных булевых функций}

В [5, теорема 3] доказано следующее утверждение: при любом виде записи слабо положительных (слабо отрицательных) функций в любом конечном алфавите $A$ для любого полинома $p(n)$ можно указать такое $n_{0} \in N$ (которое зависит от вида записи, алфавита $A$ и от полинома $p(n)$ ), что при $n \geqslant n_{0}$ существуют функции из $W P_{n}\left(W N_{n}\right)$, длина записи которых превышает $p(n)$.

Для любой мультиаффинной или биюнктивной функции $f\left(x_{1}, \ldots, x_{n}\right)$ (в отличие от слабо положительных и слабо отрицательных) существует короткая запись (запись не длиннее $p(n)$, где $p$ - некоторый фиксированный полином): такой короткой записью для этих функций является приведенное представление. 
Поэтому актуальной является задача построения слабо положительных и слабо отрицательных функций, имеющих короткую запись. При этом особый интерес представляют равновероятные функции.

Функция $f\left(x_{1}, \ldots, x_{n}\right)$ называется дважды слабо положительной ([6, с. 373]), если $f \in W P, \bar{f} \in W P$. Множество дважды слабо положительных функций обозначим $2 W P$.

Теорема 4. Пусть

$$
f\left(x_{1}, \ldots, x_{k}, y_{1}, \ldots, y_{t}\right) \equiv f_{1}\left(x_{1}, \ldots, x_{k}\right) \oplus f_{2}\left(y_{1}, \ldots, y_{t}\right),
$$

где $k \geqslant 1, t \geqslant 1$, и функиия $f$ существенно зависит от всех своих переменных. Тогда $f \in W P$, если и только если

$$
f_{1}\left(x_{1}, \ldots, x_{k}\right) \equiv \bar{x}_{1} \cdot \ldots \cdot \bar{x}_{k}, \quad f_{2}\left(y_{1}, \ldots, y_{t}\right) \equiv y_{1} \vee \ldots \vee y_{t}
$$

или

$$
f_{1}\left(x_{1}, \ldots, x_{k}\right) \equiv x_{1} \vee \ldots \vee x_{k}, \quad f_{2}\left(y_{1}, \ldots, y_{t}\right) \equiv \bar{y}_{1} \cdot \ldots \cdot \bar{y}_{t} .
$$

Замечание 2. Случаи (11) и (12) соответствуют одной и той же функции $f$. Действительно, пусть $x=\left(x_{1}, \ldots, x_{k}\right), y=\left(y_{1}, \ldots, y_{t}\right)$, тогда для (11) имеем:

$$
\begin{aligned}
& f(x, y) \equiv \bar{x}_{1} \cdot \ldots \cdot \bar{x}_{k} \oplus y_{1} \vee \ldots \vee y_{t} \equiv \\
& \equiv\left(f_{1}(x) \oplus 1\right) \oplus f_{2}(y) \oplus 1 \equiv x_{1} \vee \ldots \vee x_{k} \oplus y_{1} \vee \ldots \vee y_{t} \oplus 1 \equiv \\
& \equiv f_{1}(x) \oplus\left(f_{2}(y) \oplus 1\right) \oplus 1 \equiv \bar{x}_{1} \cdot \ldots \cdot \bar{x}_{k} \oplus \bar{y}_{1} \cdot \ldots \cdot \bar{y}_{t} \oplus 1 \equiv \\
& \equiv\left(f_{1}(x) \oplus 1\right) \oplus\left(f_{2}(y) \oplus 1\right) \equiv x_{1} \vee \ldots \vee x_{k} \oplus \bar{y}_{1} \cdot \ldots \cdot \bar{y}_{t} .
\end{aligned}
$$

Доказательство. Доказательство теоремы 5 проведем по следующему плану. Вначале покажем, что если $f \in W P$, то $f_{1} \in W P, f_{2} \in W P$. Более того, функции $f_{1}$ и $f_{2}$ оказываются дважды слабо положительными. Далее, используя свойства дважды слабо положительных функций, покажем, что функция $f$ имеет вид (11) или (12).

Пусть функция $f$ имеет вид (10) и $f \in W P$. Так как $f \in W P$, то любая функция, получаемая из $f$ фиксацией переменных, тоже слабо положительна. Предположим, что $f_{1} \notin W P$, тогда существуют такие векторы $\alpha, \beta \in V_{k}$, что $f_{1}(\alpha)=f_{1}(\beta)=1, \quad f_{1}(\alpha \vee \beta)=0$. Так как $f_{2}$ существенно зависит от своих переменных, то существует такой вектор $\gamma \in V_{t}$, что $f_{2}(\gamma)=0$. Тогда для векторов $(\alpha, \gamma),(\beta, \gamma) \in V_{k+t}$ справедливы равенства

$$
f(\alpha, \gamma)=1, f(\beta, \gamma)=1, f(\alpha \vee \beta, \gamma)=0,
$$


и по критерию слабой положительности $f \notin W P$. Значит, $f_{1} \notin W P$.

Осталось рассмотреть случай, когда $f \in W P, f_{1} \in W P, f_{2} \in W P$. Пусть $\delta=\left(\delta_{1}, \ldots, \delta_{t}\right) \in V_{t}$. Под записью вида $f(x, \delta)$ понимаем функцию $f\left(x_{1}, \ldots, x_{k}, \delta_{1}, \ldots, \delta_{t}\right)$. Существует такой вектор $\delta=\left(\delta_{1}, \ldots, \delta_{t}\right) \in V_{t}$, что $f_{2}(\delta)=1$. Функция $f(x, \delta)$ имеет вид $f(x, \delta) \equiv f_{1}(x) \oplus 1 \in W P$. Следовательно, $f_{1} \in 2 W P$ (аналогично доказывается, что $f_{2} \in 2 W P$ ).

Далее потребуются результаты из [6, с. 378-381] о строении дважды слабо положительных функций.

Пусть $a$ и $b$ - целые неотрицательные числа. Определим булеву функцию $h_{b}\left(x_{1}, \ldots, x_{a}, x_{a+1}, \ldots, x_{a+b}\right)$ по правилу

$$
\begin{gathered}
h_{b}\left(x_{1}, \ldots, x_{a}, x_{a+1}, \ldots, x_{a+b}\right) \equiv \\
\equiv \begin{cases}1, & \text { если } a=b=0, \\
\bar{x}_{1} \cdot \ldots \bar{x}_{b}, & \text { если } a=0, b>0, \\
x_{1} \vee \ldots \vee x_{a}, & \text { если } a>0, b=0, \\
\wedge_{i=1}^{b}\left(x_{1} \vee \ldots \vee x_{a} \vee \bar{x}_{a+i}\right), & \text { если } a>0, b>0 .\end{cases}
\end{gathered}
$$

В [6] доказано, что для любой дважды слабо положительной функции $f_{1}\left(x_{1}, \ldots, x_{k}\right), k \geqslant 1$, существенно зависящей от всех переменных $x_{1}, \ldots, x_{k}$, имеется единственное представление вида

$$
\begin{gathered}
f_{1} \equiv h_{d_{1}}\left(x_{s_{1}}, \ldots, x_{s_{d_{1}}}\right) \wedge h_{d_{3}-d_{2}}\left(x_{s_{d_{1}+1}}, \ldots, x_{s_{d_{2}}}, x_{s_{d_{2}+1}}, \ldots, x_{s_{d_{3}}}\right) \wedge \\
\wedge h_{d_{5}-d_{4}}\left(x_{s_{d_{1}+1}}, \ldots, x_{s_{d_{2}}}, x_{s_{d_{3}+1}}, \ldots, x_{s_{d_{4}}}, x_{s_{d_{4}+1}}, \ldots, x_{s_{d_{5}}}\right) \wedge \\
\ldots \ldots \ldots \ldots \ldots \ldots \ldots \\
\wedge h_{k-d_{2 m}}\left(x_{s_{d_{1}+1}}, \ldots, x_{s_{d_{2}}}, \ldots, x_{s_{d_{2 m}+1}}, \ldots, x_{s_{k}}\right)
\end{gathered}
$$

где $m \geqslant 1, d_{1}=k$ или $0 \leqslant d_{1}<d_{2}<\ldots<d_{2 m-1}<d_{2 m} \leqslant k, s_{1}<\ldots<$ $s_{d_{1}}, s_{d_{1}+1}<\ldots<s_{d_{2}}, s_{d_{2 m}+1}<\ldots<s_{k}$.

Пусть функция $f_{1}$ из (10) имеет вид (14), а функция $f_{2}$ из (10) имеет вид

$$
\begin{gathered}
f_{2} \equiv h_{e_{1}}\left(y_{w_{1}}, \ldots, y_{w_{e_{1}}}\right) \wedge h_{e_{3}-e_{2}}\left(y_{w_{e_{1}+1}}, \ldots, y_{w_{e_{2}}}, y_{w_{e_{2}+1}}, \ldots, y_{w_{e_{3}}}\right) \wedge \\
\wedge h_{e_{5}-e_{4}}\left(y_{w_{e_{1}+1}}, \ldots, y_{w_{e_{2}}}, y_{w_{e_{3}+1}}, \ldots, y_{w_{e_{4}}}, y_{w_{e_{4}+1}}, \ldots, y_{w_{e_{5}}}\right) \wedge \\
\ldots \ldots \ldots \ldots \ldots \ldots . . . \\
\wedge h_{t-e_{2 p}}\left(y_{w_{e_{1}+1}}, \ldots, y_{w_{e_{2}}}, \ldots, y_{w_{e_{2}+1}}, \ldots, y_{w_{t}}\right)
\end{gathered}
$$


где $p \geqslant 1, e_{1}=t$ или $0 \leqslant e_{1} \leqslant e_{2}<\ldots<e_{2 p-1}<e_{2 p} \leqslant t, w_{1}<\ldots<w_{e_{1}}$, $w_{e_{1}+1}<\ldots<w_{e_{2}}, w_{e_{2 p}+1}<\ldots<w_{t}$.

Заметим, что в записи (14) и (15) каждое переменное входит только в нулевой степени или только в первой степени (литералы вида $x_{i}$ ). Пусть в $f_{1}$ входят переменные в разных степенях. Будем считать, что $x_{i}$ входит в (14) в первой степени, а $x_{j}-$ в нулевой степени. Тогда некоторой фиксацией переменных в $f_{1}$ можем получить подфункцию $f_{1}^{\prime} \equiv x_{i} \vee \bar{x}_{j}$ или $f_{1}^{\prime} \equiv x_{i} \cdot \bar{x}_{j}$. Некоторой фиксацией переменных в $f_{2}$ можем получить подфункцию $f^{\prime} \equiv y_{1}^{\nu}$. При этих фиксациях получим одну из двух подфункций функции $f$ :

$$
f^{\prime} \equiv\left(x_{i} \vee \bar{x}_{j}\right) \oplus y_{1}^{\nu} \text { или } f^{\prime} \equiv x_{i} \bar{x}_{j} \oplus y_{1}^{\nu}
$$

Нетрудно показать, что в зависимости от вида подфункции (16) всегда можем так зафиксировать $x_{i}$ или $x_{j}$, что получим функцию $f^{\prime \prime} \equiv x_{l} \oplus y_{1}$ (где $l \in\{i, j\})$. Эта функция не является слабо положительной, поэтому в (14) (и аналогично в (15)) не могут входить переменные в разных степенях.

Далее считаем, что в функции $f_{1}, f_{2}$ все переменные входят в одинаковых степенях. Если в (14) все переменные входят только в нулевой степени, то $f_{1} \equiv \bar{x}_{1} \cdot \ldots \cdot \bar{x}_{k}$, а если в (14) все переменные входят только в первой степени, то $f_{1} \equiv x_{1} \vee \ldots \vee x_{k}$. Если в (14) и (15) все переменные входят только в первых степенях, то

$$
f \equiv x_{1} \vee \ldots \vee x_{k} \oplus y_{1} \vee \ldots \vee y_{t}
$$

Такая функция $f$ не слабо положительная, поскольку $f\left(1_{k}, 0_{t}\right)=f\left(0_{k}, 1_{t}\right)=$ $1, f\left(1_{k}, 1_{t}\right)=0$.

Так как

$$
\left(x_{1} \vee \ldots \vee x_{k}\right) \oplus\left(y_{1} \vee \ldots \vee y_{t}\right) \equiv\left(\bar{x}_{1} \cdot \ldots \cdot \bar{x}_{k}\right) \oplus\left(\bar{y}_{1} \cdot \ldots \cdot \bar{y}_{t}\right)
$$

то в (14) и (15) все переменные не могут входить только в нулевых степенях.

Осталось рассмотреть только случаи (11) и (12). Пусть выполняется (11); покажем, что $f \in W P$. Выберем такие векторы $\alpha, \beta \in V_{k}, \delta, \varepsilon \in V_{t}$, что $f(\alpha, \delta)=f(\beta, \varepsilon)=1$. Если $f_{1}(\alpha)=f_{1}(\beta)=0$, то $f_{1}(\alpha \vee \beta)=0$, $f_{2}(\delta)=f_{2}(\varepsilon)=1, \quad f_{2}(\delta \vee \varepsilon)=1$. Поэтому $f(\alpha \vee \beta, \delta \vee \varepsilon)=1$. Аналогично показывается, что свойство слабой положительности выполняется при $f_{1}(\alpha)=f_{1}(\beta)=1$ и при $f_{1}(\alpha) \neq f_{1}(\beta)$. Таким же способом рассматривается случай (12). Теорема доказана.

Следствие 1. Не сущуествует слабо положительных функиий $f\left(x_{1}, \ldots, x_{k}, y_{1}, \ldots, y_{t}, z_{1}, \ldots, z_{l}\right)$, где $k \geqslant 1, t \geqslant 1, l \geqslant 1$, существенно 
зависящих от всех своих переменных и таких, что

$$
f(x, y, z) \equiv f_{1}(x) \oplus f_{2}(y) \oplus f_{3}(z) .
$$

Доказательство. Обозначим $f_{12}(x, y) \equiv f_{1}(x) \oplus f_{2}(y)$, при этом $f(x, y, z) \equiv$ $f_{12}(x, y) \oplus f_{3}(z)$. По теореме 5

$$
f_{12}(x, y) \equiv \bar{x}_{1} \cdot \ldots \cdot \bar{x}_{k} \cdot \bar{y}_{1} \cdot \ldots \cdot \bar{y}_{t} \text { или } f_{12}(x, y) \equiv x_{1} \vee \ldots \vee x_{k} \vee y_{1} \vee \ldots \vee y_{t} \text {. }
$$

Существует такой вектор $\alpha \in V_{l}$, что $f_{3}(\alpha)=0$. Для функции $f(x, y, \alpha) \equiv$ $f_{12}(x, y)$ должно выполняться (11) или (12), но при этом невозможно (17). Следствие доказано.

Теорема 5. Пусть

$$
g\left(x_{1}, \ldots, x_{k}, y_{1}, \ldots, y_{t}\right) \equiv g_{1}\left(x_{1}, \ldots, x_{k}\right) \oplus g_{2}\left(y_{1}, \ldots, y_{t}\right),
$$

где $k \geqslant 1, t \geqslant 1$, и функиия $g$ существенно зависит от всех своих переменных. Тогда $g \in W N$, если и только если

$$
g_{1}\left(x_{1}, \ldots, x_{k}\right) \equiv x_{1} \cdot \ldots \cdot x_{k}, g_{2}\left(y_{1}, \ldots, y_{t}\right) \equiv \bar{y}_{1} \vee \ldots \vee \bar{y}_{t}
$$

или

$$
g_{1}\left(x_{1}, \ldots, x_{k}\right) \equiv \bar{x}_{1} \vee \ldots \vee \bar{x}_{k},{ }_{2}\left(y_{1}, \ldots, y_{t}\right) \equiv y_{1} \cdot \ldots \cdot y_{t}
$$

Доказательство. Очевидно, что

$$
g\left(x_{1}, \ldots, x_{k}, y_{1}, \ldots, y_{t}\right) \in W N \Leftrightarrow g\left(\bar{x}_{1}, \ldots, \bar{x}_{k}, \bar{y}_{1}, \ldots, \bar{y}_{t}\right) \in W P .
$$

Возможные случаи $g\left(\bar{x}_{1}, \ldots, \bar{x}_{k}, \bar{y}_{1}, \ldots, \bar{y}_{t}\right) \in W P$ описаны в теореме 5 , откуда следует справедливость теоремы 6.

Лемма 2. Вес функиии (13) равен

$$
\left\|f\left(x_{1}, \ldots, x_{k}, y_{1}, \ldots, y_{t}\right)\right\|=2^{k+t}-2^{k}-2^{t}+2 .
$$

Доказательство. Легко видеть, что функция $f$ равна 1 , если

1) $\left(x_{1}, \ldots, x_{k}, y_{1}, \ldots, y_{t}\right)=0_{k+t}$ или

2) $\left(x_{1}, \ldots, x_{k}\right) \neq 0_{k}$ и $\left(y_{1}, \ldots, y_{t}\right) \neq 0_{t}$. 
Поэтому $\|f\|=1+\left(2^{k}-1\right)\left(2^{t}-1\right)=2^{k+t}-2^{k}-2^{t}+2$.

Следствие 2. Функиия вида (13) является равновероятной, если и только если $k=1$ или $t=1$.

Доказательство. Пусть $t=1$, тогда $\|f\|=2^{(k+1)-1}$. Заметим, что в случае $k=1$ или $t=1$ функция $f$ является равновероятной как функция, равная сумме по модулю 2 двух функций, зависящих от разных переменных, причем одна из этих функций равновероятная.

Предположим, что $k \geqslant 2, t \geqslant 2$. Тогда $2^{k-1}+2^{t-1}-1-$ нечетное число и $2^{k-1}+2^{t-1}-1 \neq 2^{k+t-2}, \quad 2^{k}+2^{t}-2 \neq 2^{k+t-1}$. Поэтому функция $f$ не является равновероятной.

Пусть $k+t=n(k \geqslant 1, t \geqslant 1)$ фиксировано. Символом $R_{n}$ обозначим множество функций, которые могут быть получены из всех функций вида (13) перестановкой переменных, $r_{n}=\left|R_{n}\right|$.

Разрядом называется класс эквивалентности булевых функций относительно перестановки переменных.

Лемма 3. Число $r_{n}$ находится по формуле

$$
r_{n}=2^{n-1}-1 .
$$

Доказательство. Множество $R_{n}$ разбивается на разряды

$$
R_{n}=\left[f^{(1)}\right]_{\left\langle S_{n}\right\rangle} \cup \ldots \cup\left[f^{(u)}\right]_{\left\langle S_{n}\right\rangle} .
$$

В каждом разряде из $R_{n}$ можем выбрать представитель вида (15), для которого $k \leqslant t$, поэтому число разрядов $u$ равно $u=\left\lfloor{ }^{n} / 2\right\rfloor$, где $\lfloor n / 2\rfloor-$ целая часть числа $n / 2$. Далее считаем, что в представителях классов (см. (19)) индекс $i$ равен соответствующему $k$.

Если $k<t$, то $\left|\left[f^{(k)}\right]_{\left\langle S_{n}\right\rangle}\right|=\left(\begin{array}{l}n \\ k\end{array}\right)$. В случае $k=t$ нетрудно показать, что $\left|\left[f^{(n / 2)}\right]_{\left\langle S_{n}\right\rangle}\right|=1 / 2 \cdot\left(\begin{array}{c}n \\ n / 2\end{array}\right)$. Отсюда следует (18).

Очевидна следующая теорема.

Теорема 6. Пусть $f_{1}\left(x_{1}, \ldots, x_{k}\right) \not \equiv 0, f_{2}\left(y_{1}, \ldots, y_{t}\right) \not \equiv 0$, тогда

$$
f\left(x_{1}, \ldots, x_{k}, y_{1}, \ldots, y_{t}\right) \equiv f_{1}\left(x_{1}, \ldots, x_{k}\right) \cdot f_{2}\left(y_{1}, \ldots, y_{t}\right) \in W P,
$$


если и только если $f_{1}(x) \in W P, f_{2}(y) \in W P$.

Символом $M$ обозначим множество монотонных булевых функций.

Теорема 7. Пусть

$$
f\left(x_{1}, \ldots, x_{k}, y_{1}, \ldots, y_{t}\right) \equiv f_{1}\left(x_{1}, \ldots, x_{k}\right) \vee f_{2}\left(y_{1}, \ldots, y_{t}\right),
$$

$f_{1} \not \equiv 1, f_{2} \not \equiv 1$. Тогда $f \in W P$, если и только если

$$
f_{1} \in W P, f_{2} \in M \text { или } f_{1} \in M, f_{2} \in W P .
$$

Доказательство. Предположим, что $f_{1} \in W P, f_{2} \in M$, что функция $f_{1}$ имеет приведенное представление (3), а $f_{2}$ можно записать в виде

$$
f_{2} \equiv \bigwedge_{j=1}^{q}\left(y_{r_{j 1}} \vee \ldots \vee y_{r_{j_{j}}}\right) .
$$

Для любых булевых функций $g_{i}$ и $h_{j}$ справедливо тождество

$$
\left(\hat{i}_{i} g_{i}\right) \vee\left({ }_{j} h_{j}\right) \equiv \bigwedge_{i} \wedge_{j}\left(g_{i} \vee h_{j}\right)
$$

Из приведенных рассуждений следует, что $f \in W P$.

Пусть теперь $f \in W P$. Будем считать, что $f_{1}$ и $f_{2}$ записаны сокращенными КНФ. Нетрудно видеть, что если при этом записать $f$ в соответствии с (3), то получится сокращенная КНФ функции $f$. Отсюда вытекает, что $f_{1} \in W P, f_{2} \in M$ или $f_{1} \in M, f_{2} \in W P$.

Автор выражает признательность А. В. Тарасову за ценные замечания.

\section{Список литературы}

[1] Schaefer T., "Complexity of satisfiability problems", Proc. 10 Annu. ACM Symp. Theory Comput. Machin. (1978), 216-226.

[2] Гизунов С.А., Носов В. А., “О классификации всех булевых функций четырех переменных по классам Шеффера”, Обозрение прикл. и промышл. матем., 2 :3 (1995), 440-467.

[3] Глухов М. М., Шишков А. Б., Математическая логика. Дискретные функиии. Теория алгоритмов, СПб. : Лань, 2012.

[4] Тарасов А. В., “О свойствах функций, представленных в виде 2-КНФ”, Дискретная математика, $13: 4$ (2001), 99-115.

[5] Горшков С. П., “О некоторых свойствах слабо положительных и слабо отрицательных булевых функций”, Прикладная дискретная математика, 20 (июнь 2013), 5-13.

[6] Горшков С. П., "Применение теории NP-полных задач для оценки сложности решения систем булевых уравнений”, Обозрение прикл. и промышл. матем., 2 :3 (1995), 325-398. 